\title{
INVESTIGATION OF THE STABILITY OF COMMERCIAL NEUTRON PROBES
}

\author{
H. Hoedlmoser*, G. Butterweck, Ch. Schuler and S. Mayer \\ Paul Scherrer Institut, 5232 Villigen PSI, Switzerland \\ *Corresponding author: herbert.hoedlmoser@psi.ch
}

Received January 4 2012, revised March 26 2012, accepted April 12012

\begin{abstract}
At the Paul Scherrer Institute's Calibration Laboratory, neutron reference fields are provided for the calibration of ambient and personal dose equivalent (rate) metres and passive dosemeters. To ensure traceability to the standards of the Physikalisch-Technische Bundesanstalt (PTB) in Germany, the neutron fields are characterised by means of a PTB-calibrated Berthold LB6411 neutron probe which is used as a secondary standard. The LB6411 detector suffers from an unstable, increasing dose rate reading in the order of up to $+5 \%$ (according to the manufacturers, this is due to a charging effect in the ${ }^{3} \mathrm{He}$ proportional counter). In a calibration, this instability is usually corrected for based on the reading obtained with a test source. In this work, the instability was investigated by means of measurements under irradiation with ambient dose equivalent rates up to $24 \mathrm{mSv} \mathrm{h}^{-1}$ for up to $20 \mathrm{~h}$ and compared with the behaviour of an LB6419 and a Thermo Wendi-2 probe. The reading of the instruments was found to reach a plateau, e.g. it becomes stable after $\sim 90$ min during irradiation with 10 $\mathrm{mSv} \mathrm{h}^{-1}$ neutrons. The plateau is reached faster for higher dose rates. This supports the interpretation as a charging effect in the proportional counter. The effect could also be duplicated in an irradiation with photons from a ${ }^{137} \mathrm{Cs}$ source. The decay time of the accumulated charge was found to be very long, i.e. the instrument showed a stable reproducible reading for up to 6 h after the plateau was reached. From these observations, a conditioning procedure was derived which ensures a stable operation of the instrument after an irradiation of the instrument preceding its use in the reference measurements.
\end{abstract}

\section{INTRODUCTION}

The Paul Scherrer Institute's (PSI's) Calibration Laboratory uses a Berthold LB6411 neutron detector as a secondary standard to characterise the neutron fields of a ${ }^{241} \mathrm{Am}-\mathrm{Be}$ and $\mathrm{a}{ }^{252} \mathrm{Cf}$ calibration source as described in references ${ }^{(1-3)}$. This neutron detector is a ${ }^{3} \mathrm{He}$ proportional counter combined with an optimised spherical moderator ${ }^{(4)}$. The PSI instrument is calibrated at the Physikalisch-Technische Bundesanstalt (PTB) in Braunschweig every 5 y. A known issue with LB6411 neutron probes is their signal drift during continuous irradiation. According to information from the manufacturer ${ }^{(5)}$, the instability of the LB6411 is attributed to a charge accumulation on insulating surfaces in the ${ }^{3} \mathrm{He}$ proportional counter of the device. The accumulated charge modifies the electrical field in the counter and lowers the effective discriminator level above which pulses are counted as neutrons, thus increasing the count rate. After irradiation, the charge slowly dissipates, roughly with the time constant corresponding to the resistivity times capacitance value of the counter. In the past, Berthold has attempted to correct the problem by means of an additional guard electrode inside the proportional counter. However, this remedy was not successful due to gas leakage problems with the additional electrical feed-through and the attempt was abandoned, as the signal drift is small enough to be ignored for the standard use of the instrument in the field ${ }^{(5)}$. As a consequence of this signal drift, the PTB calibration is only valid in connection with a certain reading with a test source (Amersham 370 $\mathrm{MBq}{ }^{241} \mathrm{Am}-\mathrm{Be}, 2.4 \mathrm{E} 4 \mathrm{n} / \mathrm{s}, 28$ November 1980) which was shipped to PTB together with the detector. The reading with this source was recorded during the calibration at PTB. A deviation in the reading produced by the test source needs to be taken into account by means of a correction factor $c_{\mathrm{S}}$ for stability. In this work, the effect was investigated in several long-term measurements with varying dose rates and compared with the behaviour of a Berthold LB6419 $9^{(6)}$ and a Thermo Wendi-2 ${ }^{(7)}$ detector which both use similar ${ }^{3} \mathrm{He}$ proportional counters albeit with a different moderator design. All three instruments were found to reach a plateau after continued irradiation.

\section{LB6411 DETECTOR AND PTB CALIBRATION}

The LB6411 neutron detector of the PSI Calibration Laboratory (SNr. 1269) is used with an UMO LB123 (SNr. 3090) dose meter, which is programmed with the factory calibration factor of 1.27 $\mu \mathrm{Sv} \mathrm{h}{ }^{-1} \mathrm{cps}^{-1}$ and displays a reading $M$ for the ambient dose equivalent rate $\mathrm{d} H^{*}(10) / \mathrm{d} t$. According to the PTB calibration certificate, this reading is corrected by means of a dimensionless calibration factor $N$ and correction factors for linearity $\left(c_{\mathrm{L}}\right)$, stability $\left(c_{\mathrm{S}}\right)$ and a field-specific correction $\left(c_{\mathrm{F}}\right)$ for 
radiation quality. A final correction that needs to be applied is a geometry correction $\left(c_{\mathrm{G}}\right)$ that takes the inhomogeneity of the field for small distances from the source into account ${ }^{(8)}$. In summary, the ambient dose equivalent rate $\mathrm{d} H^{*}(10) / \mathrm{d} t$ is calculated as $\mathrm{d} H^{*}(10) / \mathrm{d} t=M \cdot N \cdot c_{\mathrm{L}} \cdot c_{\mathrm{F}} \cdot c_{\mathrm{S}} \cdot c_{\mathrm{G}}$

\section{MEASURED SIGNAL DRIFT}

Measurements of the instrument stability were carried out at the PSI Calibration Laboratory with the ${ }^{252} \mathrm{Cf}$ source for time periods of up to $20 \mathrm{~h}$. The observed signal drift is an increase of the reading of the instrument in the order of $\sim 3-8 \%$ depending on the irradiation history before the measurement. An example for the measured effect can be found in Figure 1. The effect has also been observed with a Thermo Wendi-2 (SN 18893) and a Berthold LB6419 ( $\mathrm{SNr}$ 38), both instruments that use a similar ${ }^{3} \mathrm{He}$ proportional counter.

For the investigated LB6411, the effect is not related to any warm up phase of the instrument, as the device is always operated for a minimum of 30 min before any irradiations or measurements take place. As can be seen in Figure 1, the reading of the instrument increases for a certain time and then reaches a plateau. The effect is only observed during irradiation and the time it takes to arrive at the plateau is correlated with the dose rate during the irradiation (Figure 2). For higher dose rates, the plateau is achieved sooner, which supports the assumption of a charging effect due to the free charges produced in the irradiation.

\section{STABILITY OF THE PLATEAU READING}

Once the reading of the LB6411 has achieved a plateau, the instrument can be considered 'conditioned' and in this condition operates in a stable manner. Short interruptions of the irradiation for up to $6 \mathrm{~h}$ and changes in the applied dose rate do not cause the instrument to lose this conditioning.

Figure 3 shows the reading of the LB6411 during a dedicated long-term test. In the first part of the test (Figure 3a), the instrument was stabilised at a dose rate of $\sim 1 \mathrm{mSv} \mathrm{h}^{-1}$. Then the irradiation was interrupted for $6 \mathrm{~h}$. After resuming the irradiation, the level of the plateau was reproduced. Subsequently, the dose rate was increased in two steps up to $\sim 24 \mathrm{mSv} \mathrm{h}^{-1}$. After each increase, the instrument immediately showed a stable reading (Figure $3 b$ ). Then the irradiation was stopped for $\sim 20 \mathrm{~h}$ during which the LB6411 was switched off. After the interruption, the instrument had to be stabilised again at the high-dose rate $\left(24 \mathrm{mSv} \mathrm{h}^{-1}\right)$. Therefore, it is assumed that the accumulated charge decays with a relatively large time constant. After the renewed stabilisation, the dose rate was lowered to the same levels as in the previous test and the old levels could be reproduced with immediately stable readings (Figure 3c). The time constant of the charge depletion in the LB6411 is not known exactly, as these investigations are extremely time-consuming. However, from the observations presented above, it is clear that the reading of an unconditioned LB6411 depends on the previous irradiation history and on the time that has passed since the last use of the instrument. For example, in several conditioning experiments such as shown in Figure 2, it was

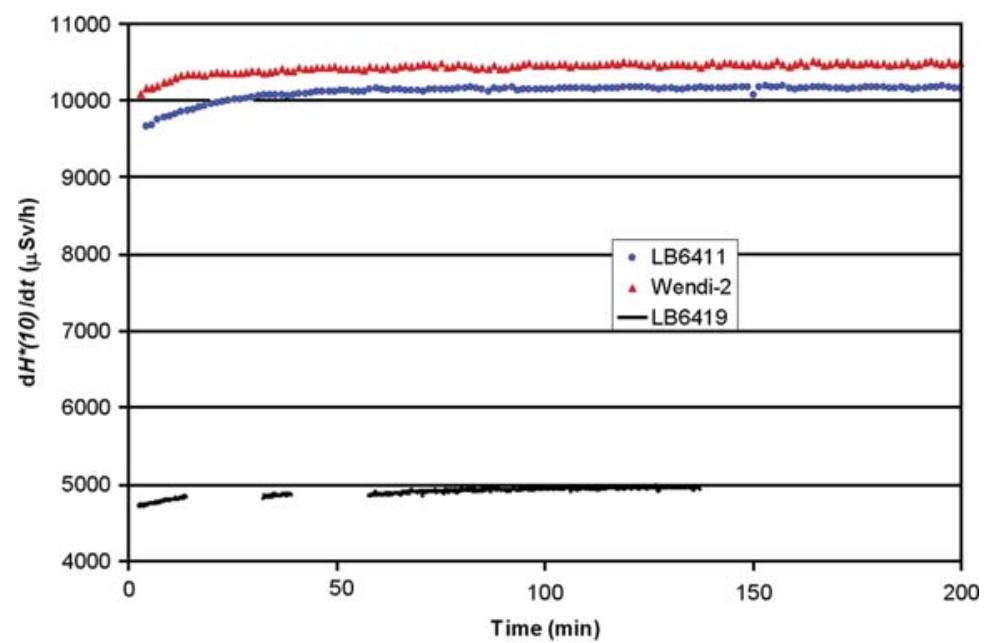

Figure 1. Signal drift at the beginning of an irradiation with a ${ }^{252} \mathrm{Cf}$ source. The evolution of the signal from a LB6411, a LB6419 and a Wendi-2 are compared. For the LB6419, the test was carried out at a lower level of ambient dose equivalent rate. 


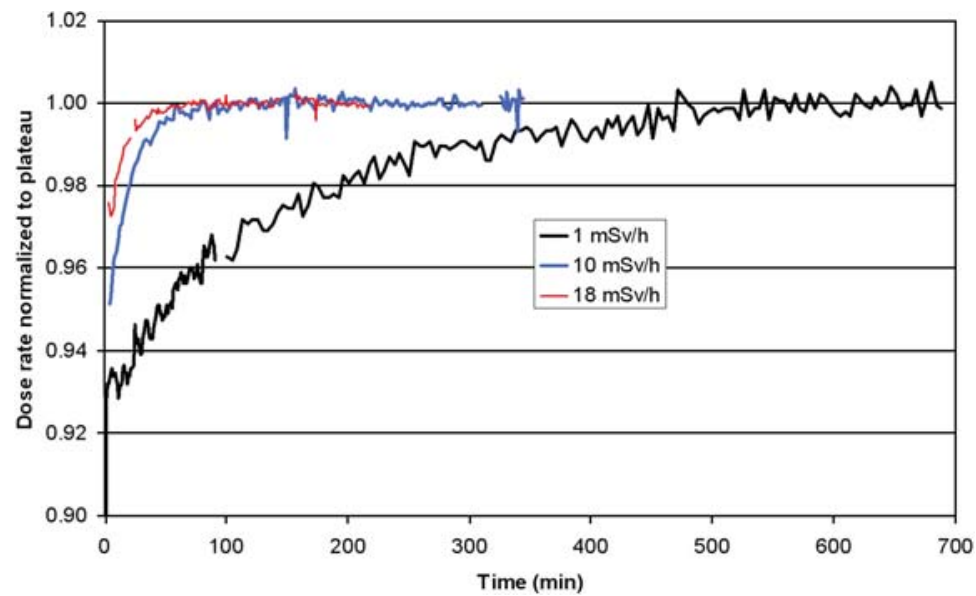

Figure 2. With increasing dose rate, the stabilisation of the instrument reading of the LB6411 is achieved faster indicating a charge up effect. The different dose rates have been realised by means of a ${ }^{252} \mathrm{Cf}$ and an ${ }^{241} \mathrm{Am}-\mathrm{Be}$ source.
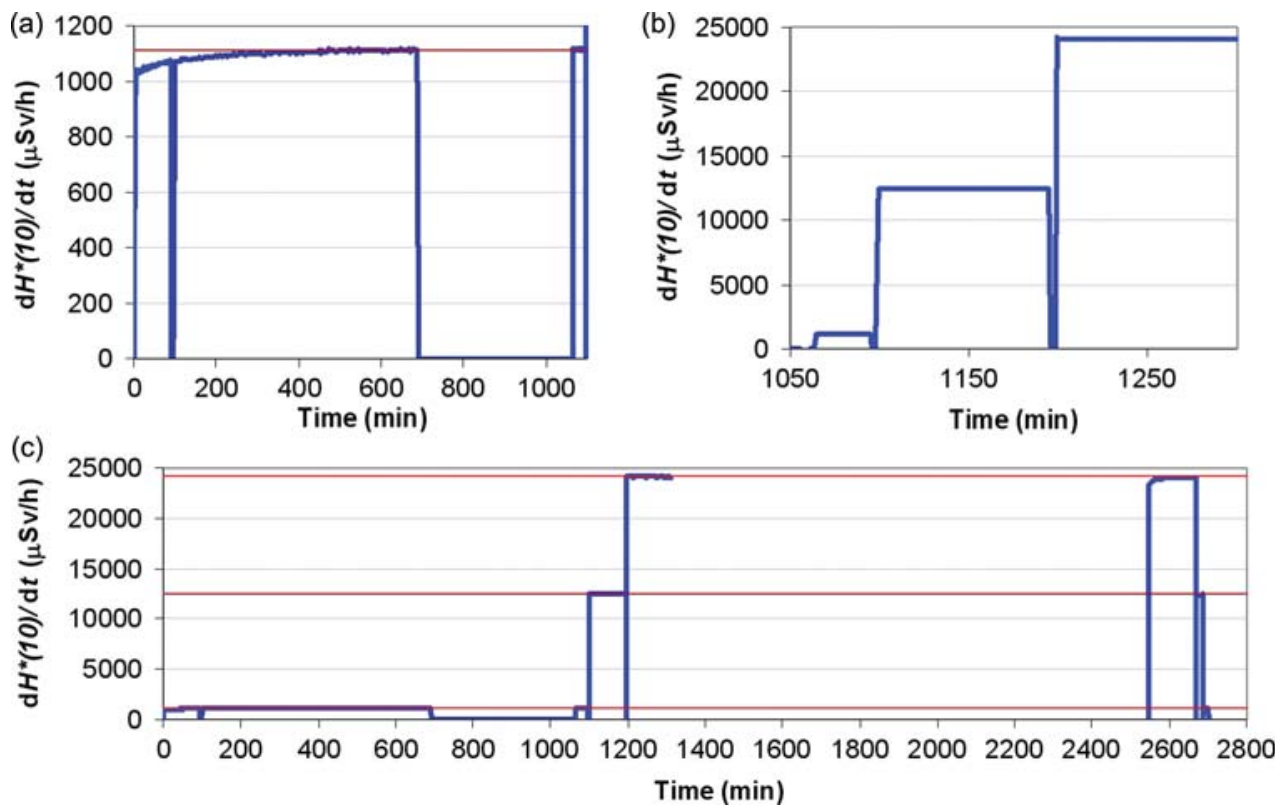

Figure 3. The observation of the LB6411 reading during a long-term test with the ${ }^{252} \mathrm{Cf}$ source. The figures (a) and (b) are extracts from the overall plot in (c). See text for explanations.

observed that the initial level before conditioning depends on how much time has passed since the last use of the instrument, whereas the final level of the plateau remains unchanged. This has also been verified in the conditioning experiments combined with test source measurements, which are discussed in the following section.

\section{CORRECTION $C_{\mathrm{S}}$ FOR A CONDITIONED INSTRUMENT}

For its use as a secondary standard, the LB6411 is regularly shipped to PTB for calibration together with an ${ }^{241} \mathrm{Am}-\mathrm{Be}$ test source. Due to the instability of the instrument, it is necessary to normalise all measurements with the LB6411 to the reading 


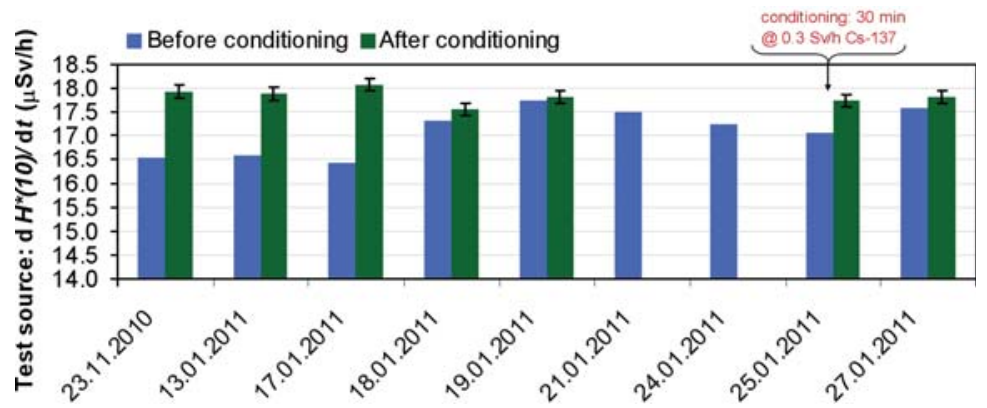

Figure 4. Test source reading before and after conditioning under neutron irradiation. The level after conditioning is reproducible with a relative standard uncertainty of $1.4 \%$. The conditioning is lost slowly during the following days, and it can also be achieved in an irradiation with photons. This figure appears in colour in the online version of Radiation Protection Dosimetry.

$M_{\text {Am-BeSource }}$ with the test source relative to the reading $M_{\mathrm{Am}-\mathrm{BeSourcePTB}}$ measured at PTB. The reading $M_{\mathrm{Am}-\mathrm{BeSourcePTB}}$ is obtained during calibration at PTB in a documented and reproducible counting geometry and monitored throughout the calibration. When the instrument is subsequently used at PSI, a correction factor $c_{\mathrm{S}}=M_{\mathrm{Am}-\mathrm{BeSourcePTB}} / M_{\mathrm{Am}-\text { BeSource }}$ has to be applied with $M_{\mathrm{Am}-\mathrm{BeSourcePTB}}=16.19 \pm 0.19 \mu \mathrm{Sv} \mathrm{h}^{-1}(k=2)$. For the conditioned (=stabilised) LB6411, the reading $M_{\mathrm{Am} \text {-BeSource }}$ was confirmed to be reproducible in a series of conditioning experiments. In these tests, the reading with the test source was taken before and after the conditioning procedure as a mean value of $\sim 200$ rate measurements recorded in the LB6411 for approximately half an hour. The results are shown in Figure 4. The conditioning could also be achieved by an irradiation with high doses of photons, e.g. $30 \mathrm{~min}$ with $0.3 \mathrm{~Sv} \mathrm{~h}^{-1}$ photons from a ${ }^{37} \mathrm{Cs}$ source. The average value for the reading with the test source after conditioning is $M_{\mathrm{Am}-\text { BeSource }}=$ $17.38 \pm 0.13 \mu \mathrm{Sv} \mathrm{h}^{-1}(k=2)$ with the standard uncertainty derived from the seven individual results. The given values are net dose rates. A background dose rate of $0.45 \mu \mathrm{Sv} \mathrm{h} \mathrm{h}^{-1}$, which was obtained from a long-term $(15 \mathrm{~h})$ dose measurement, has been subtracted from the gross rate. The resulting stability correction for the conditioned instrument is: $c_{\mathrm{S}}=M_{\mathrm{Am}-\text { BeSourcePTB }} / M_{\mathrm{Am}-\text { BeSource }}=0.932 \pm 0.013$ $(k=2)$. Provided the instrument has been conditioned as described above, this factor $c_{\mathrm{S}}$ can be used instead of repeatedly performing test source measurements and calculating new values for $c_{\mathrm{S}}$.

\section{SUMMARY AND CONCLUSIONS}

The signal drift of a Berthold LB6411 was investigated by means of long-term measurements under irradiation with ambient dose equivalent rates up to
$24 \mathrm{mSv} \mathrm{h}^{-1}$. The reading of the instrument was found to reach a plateau after an irradiation $>90$ min with $10 \mathrm{mSv} \mathrm{h}^{-1}$ neutrons from a ${ }^{252} \mathrm{Cf}$ source. A similar effect was found in a Thermo Wendi-2 and a Berthold LB6419 neutron probe. For the LB6411, the effect is assumed to be due to a charging effect in the proportional counter, as the plateau is reached faster for higher dose rates. The effect could also be duplicated by irradiating with photons from a ${ }^{137} \mathrm{Cs}$ source. The decay time of the accumulated charge was found to be very long, i.e. the instrument showed a stable reproducible reading for at least $6 \mathrm{~h}$ after the plateau was reached. From these observations, a conditioning procedure was derived which is either an irradiation with $10 \mathrm{mSv}$ $\mathrm{h}^{-1}$ of neutrons for at least $90 \mathrm{~min}$ or an irradiation with $300 \mathrm{mSv} \mathrm{h}^{-1}$ of photons from a ${ }^{137} \mathrm{Cs}$ source for at least $30 \mathrm{~min}$. This procedure ensures a stable operation of the instrument for its use as a secondary standard. With the conditioned detector, the instrument reading with the test source was found to be constant and reproducible, which allowed the determination of a more accurate correction factor $c_{s}$ for use in measurements with the conditioned instrument. For practical purposes, it can be concluded that the LB6411 is best used in the conditioned state to ensure reproducible results, especially when used as a secondary or transfer standard. At the PSI Calibration Laboratory, all measurements to establish the reference models for the available ${ }^{241} \mathrm{Am}-\mathrm{Be}$ and ${ }^{252} \mathrm{Cf}$ sources have been performed with a conditioned LB6411 ${ }^{(2,3)}$. When used routinely in the field, a conditioning may not be necessary, as the measurement uncertainties in an uncharacterised field can be expected to be much larger than the conditioning effect due to the energy response of the instrument. It should also be kept in mind that the conditioning effect can have consequences in a calibration or verification of an LB6411 probe. In a 


\section{H. HOEDLMOSER ET AL.}

verification, the instrument could pass or fail the rejection criteria depending on the current conditioning state.

\section{FUNDING}

This work was partly supported by the Swiss Federal Nuclear Safety Inspectorate (ENSI, contract N8100694).

\section{REFERENCES}

1. Hoedlmoser, H., Schuler, Ch., Butterweck, G. and Mayer, S. Characteristics of the neutron irradiation facilities of the PSI Calibration Laboratory. AIP Conf. Proc. 1412, 385-392 (2011).

2. Hoedlmoser, H., Schuler, Ch., Butterweck, G., Chetelat, N. and Mayer, S. Characterization of the ${ }^{241} \mathrm{Am}-\mathrm{Be}$ neutron source of the PSI Calibration Laboratory. PSI Technical Report TM-96-11-02, PSI (2011).
3. Hoedlmoser, H., Schuler, Ch., Butterweck, G., Karcher, A. and Mayer, S. Characterization of the ${ }^{252} \mathrm{Cf}$ and ${ }^{252} \mathrm{Cf}\left(\mathrm{D}_{2} \mathrm{O}\right)$ neutron source of the PSI Calibration Laboratory. PSI Technical Report TM-96-11-15, PSI (2011).

4. Burgkhardt, B., Fieg, G., Klett, A., Plewnia, A. and Siebert, B. R. L. The neutron fluence and $H^{*}(10)$ response of the new LB 6411 rem counter. Radiat. Prot. Dosim. 70(1-4), 361-364 (1997).

5. Private communication. A. Klett from Berthold Technologies (2012)

6. Klett, A., Leuschner, A. and Tesch, N. A dose meter for pulsed neutron fields. Radiat. Meas. 45(10), 1242-1244 (2010).

7. Olsher, R. H., Hsu, H.-H., Beverding, A., Kleck, J. H., Casson, W. H., Vasilik, D. G. and Devine, R. T. WENDI: an improved neutron rem meter. Health Phys. 79(2), 170-181 (2000).

8. International Standard ISO. Reference neutron radiations-Part 2: calibration fundamentals of radiation protection devices related to the basic quantities characterizing the radiation field. ISO 8529-2 (2000). 